\title{
Insectos del intermareal de Concepción, Chile: perspectivas para la investigación ecológica
}

\author{
Intertidal insects from Concepción, Chile: perspectives for ecological research
}

PATRICIO A. CAMUS ${ }^{1,2} \&$ RODRIGO M. BARAHONA

\author{
Departamento de Ecología Costera, Facultad de Ciencias, Universidad Católica de la Santísima \\ Concepción, Casilla 297, Concepción, Chile \\ ${ }^{1}$ Center for Advanced Studies in Ecology \& Biodiversity; \\ ${ }^{2} \mathrm{e}$-mail: pcamus@ucsc.cl
}

\begin{abstract}
RESUMEN
Los insectos son un grupo virtualmente ignorado en los estudios ecológicos de ambientes marinos litorales en Chile. Aunque se reconocen como degradadores de restos orgánicos, otros datos sugieren una participación más activa en procesos comunitarios, pero la información disponible es escasa. Con objeto de determinar los taxa de insectos presentes en el litoral de Concepción, sur de Chile (ca. 36 $40^{\prime} \mathrm{S}$ ), y su importancia ecológica potencial en el sistema, realizamos un estudio exploratorio en cuatro localidades: Caleta Tumbes, Cocholgüe, área norte de la desembocadura del río Bío-Bío y Punta de Parra. En cada localidad evaluamos la diversidad de insectos en la zona intermareal baja, media y alta, registrando el hábitat o tipo de substrato al que se encontraban asociados. Detectamos 24 taxa de insectos distribuidos en cuatro órdenes y 18 familias, incluyendo especies nativas e introducidas, parte de ellas ocasionales o casuales. La diversidad fue mayor en la zona intermareal media, particularmente en el intermareal rocoso donde hay mayor abundancia de algas. Además detectamos asociaciones recurrentes entre insectos y las especies de algas intermareales más comunes (in situ o varadas, vivas o en descomposición), usadas aparentemente como alimento o hábitat, sugiriendo un papel fundamental en el reciclamiento e intercambio de nutrientes entre el intermareal y el submareal. Algunos insectos depredadores terrestres parecen aprovechar los parches de insectos sobre algas para forrajeo, generando interacciones laterales con el intermareal. Discutimos la posible membrecía comunitaria de los insectos y las perspectivas de investigación sugeridas por este estudio.
\end{abstract}

Palabras clave: insectos marinos, intermareal, diversidad, algas.

\begin{abstract}
Insects are virtually ignored in ecological studies of littoral marine environments in Chile. Although they are known to feed on decaying organic matter, recent data suggest a more active role in community processes, but the available information is extremely scarce. With the aim to determine insect taxa occurring at the littoral zone of Concepción, Chile (ca. $36^{\circ} 40^{\prime} \mathrm{S}$ ), and their potential ecological importance in that system, we conducted an exploratory study in four localities: Caleta Tumbes, Cocholgüe, mouth (saline) of the Bío-Bío river, and Punta de Parra. At each locality, we assessed the diversity of insects in the high, mid and low intertidal zones, recording the habitat or type of substratum to which they were associated. We found 24 insect species, both native and introduced, distributed in four orders and 18 families, including midges, rover beetles, and crane flies. Some of the species were considered casual records. Diversity was higher in the mid intertidal zone, particularly in the rocky intertidal fringe where macroalgae are more abundant. We also detected recurrent associations between insects and the most common intertidal algal species (in situ or washed ashore, live or decaying), apparently used as habitat or food, suggesting a key role in the recycling and exchange of nutrients between intertidal and subtidal systems. Some terrestrial predatory insects seemed to forage consistently on patches of insects associated to decaying algae, generating lateral interaction chains with the intertidal system. We discuss the community membership of insects and the research perspectives arising from this study.
\end{abstract}

Key words: marine insects, intertidal, diversity, algae.

\section{INTRODUCCIÓN}

Los estudios realizados en hábitats intermareales marinos en general han prestado poca atención a los taxa terrestres asociados al intermareal o a sus organismos, con excepción de casos particulares en vertebrados como roedores (e.g., cricétidos y múridos; Martínez et al. 1986, Zamorano 1986, Navarrete \& Castilla 1993) o cánidos (e.g., coyotes; Rose \& Polis 1998). Los invertebrados, sin embargo, han sido virtualmente olvidados, destacando particularmente los insectos.

El poco interés de la ecología intermareal por los insectos no resulta extraño considerando que 
el grupo es poco conocido, y aparentemente poco conspicuo y poco abundante en los ambientes litorales. De hecho, de todas las especies de insectos, sólo alrededor del $3 \%$ (ca. 25.000-30.000) son acuáticas o tienen estados ontogenéticos acuáticos, y menos del $1 \%$ son marinas (ver Cheng 1976, Williams \& Feltmate 1992). Sin embargo, a nivel mundial este grupo posee 20 órdenes taxonómicos representados en ambientes marinos, con 250 a 350 especies que viven expuestas al agua de mar o en condiciones oceánicas. Muchas especies tienen o han desarrollado la capacidad de tolerar ambientes salinos (Teskey 1991), presentan rasgos fisiológicos y de historia de vida relacionados al ambiente marino litoral y los ritmos mareales (Endrab 1976b), o incluso en algunos manglares o lagunas costeras coexisten con invertebrados marinos formando comunidades de interfase (Barnes \& Hughes 1988). En general, no obstante, los insectos aún son reconocidos principalmente por su rol en la descomposición de material orgánico varado en playas (e.g., ver Teskey 1991, Raffaelli \& Hawkins 1996).

Por otra parte, si bien la presencia de insectos es un fenómeno corriente en las zonas intermareales, particularmente en la transición al sistema terrestre, es menos obvio que hayan especies propiamente marinas y/o que establezcan relaciones ecológicas importantes con componentes físicos o biológicos del ambiente intermareal. Además, los adultos en muchos casos sólo se observan en el momento de la reproducción, y el patrón de emergencia varía según la especie pudiendo seguir ritmos diarios, semi lunares o con algún componente estacional (Cheng \& Collins 1980, Neumann 1986, Soong et al. 1999). Esto hace difícil determinar la membrecía de un insecto a la comunidad intermareal, definida aquí como su calidad de residente y/o participante permanente en los flujos de materia y energía en el sistema. No obstante, la literatura (e.g., Neuman 1986, Saigusa \& Akiyama 1995) indica que mientras algunas especies (como adultos y larvas) usan de modo diferencial el gradiente vertical, otras tienden a diverger en su uso de los substratos intermareales, ya sea microhábitats o especies de algas e invertebrados. Esta variación en uso de hábitat sugiere que los insectos podrían influir simultáneamente en distintos puntos de la trama trófica y en distintos procesos biológicos de la comunidad intermareal.

En Chile, los insectos litorales aún son virtualmente desconocidos, si bien algunos estudios recientes revelan su importancia en el sistema intermareal y evidencian la necesidad de un conocimiento básico del grupo. Por ejemplo, la reproducción de las lapas del género Scurria, uno de los organismos intermareales más comunes en Chile, parece ser fuertemente afectada (hasta la castración) por larvas endoparásitas de dípteros, fenómeno que tendría gran importancia ecológica y que aparentemente es común en Chile central y norte (Uribe \& Otaíza 1996¹, C. Espoz comunicación personal). Por otra parte, Avellanal et al. (2000) y Jaramillo et al. (2000) han realizado estudios detallados en el sur de Chile sobre el coleóptero Phalerisida maculata, posiblemente uno de los principales descomponedores en el hábitat intermareal arenoso. No obstante, aún no existe mayor interés sobre el rol ecológico de los insectos en el intermareal chileno, en parte por su escaso conocimiento incluso al nivel taxonómico más elemental.

Por lo anterior, en este trabajo presentamos una evaluación preliminar de los insectos asociados a la zona intermareal en cuatro localidades del área de Concepción, centro-sur de Chile, para obtener una primera caracterización de los taxa presentes y su abundancia. Además se evaluaron las posibles asociaciones entre especies de insectos y especies marinas o tipos de hábitat intermareales. Con esta base, sugerimos algunas perspectivas sobre los posibles roles ecológicos que puedan jugar los insectos en las comunidades intermareales.

\section{MATERIALES Y MÉTODOS}

Durante diciembre de 1999 , se realizaron muestreos y capturas de insectos en la zona intermareal de cuatro localidades en la Bahía Concepción: Caleta Tumbes (36 37 '20" S, $\left.73^{\circ} 05^{\prime} 20^{\prime \prime} \mathrm{O}\right)$, Cocholgüe ( $36^{\circ} 35^{\prime} 12 \mathrm{~S}, 72^{\circ} 58^{\prime} 35^{\prime \prime}$ $\mathrm{O})$, área norte de la desembocadura del río BíoBío (36² $48^{\prime} 30^{\prime \prime} \mathrm{S}, 73^{\circ} 10^{\prime} 18^{\prime \prime} \mathrm{O}$; en condiciones no estuarinas) y Punta de Parra $\left(36^{\circ} 40^{\prime} 05^{\prime \prime} \mathrm{S}\right.$, $\left.72^{\circ} 58^{\prime} 50^{\prime \prime} \mathrm{O}\right)$. En ellas se efectuaron registros en un sector de $100 \mathrm{~m}$ de costa de zona intermareal mixta, donde alternaban hábitats rocosos y arenosos en proporción aproximadamente similar. En cada localidad se estableció un total de 15 estaciones distribuidas en grupos de cinco, cada uno asignado a tres zonas intermareales definidas como una banda de ca. $2 \mathrm{~m}$ de ancho: zona alta bajo la línea de marea más alta o inicio del sistema pro-

${ }^{1}$ URIBE R \& R OTAÍZA (1996) Primer registro de parasitismo por larvas de dípteros en patelogastrópodos de la zona intermareal rocosa. Libro de Resúmenes, Décimo Sextas Jornadas de Ciencias del Mar, Concepción, Chile. 
piamente terrestre; zona baja, inmediatamente sobre la zona de rompiente o sobre la franja del alga laminarial Lessonia nigrescens; y zona media, situada entre las anteriores. En cada zona las estaciones fueron dispuestas en paralelo a la línea de costa y separadas $20 \mathrm{~m}$ entre sí, y en cada estación se registró la presencia y número de individuos adultos de especies de insectos en un área de ca. $9 \mathrm{~m}^{2}$ con una permanencia de 30 minutos. Las capturas se efectuaron mediante mallas entomológicas y usando métodos de succión y frascos de recolección, dependiendo del tipo de insecto.

Debido al poco conocimiento de los insectos de ambientes litorales chilenos, la escasez de literatura especializada en especial sobre estados larvales, y nuestra inexperiencia disciplinaria, la determinación taxonómica fue un aspecto crítico. Los adultos capturados fueron identificados al nivel taxonómico más bajo posible, si bien con carácter preliminar, basados en la colaboración de entomólogos expertos y la literatura disponible tanto impresa (Sawada 1955, Cheng 1976, Comstock 1977, CSIRO 1979, Peña 1992, Williams \& Feltmate 1992, Merrit et al. 1996, Lizarralde 1998, Murray 1999, Murray \& Hughes 2000, Para et al. 2001) como electrónica (Evenhuis 1999, Vidal 2000). El nivel de resolución taxonómica alcanzado dependió de la disponibilidad de información y el grado de variación morfológica de los individuos capturados, y sólo el $46 \%$ de los taxa se determinó a nivel de género o especie. No obstante, la mayoría de los taxa fueron considerados especies por ser representantes únicos de una familia, excepto Staphylinidae donde se distinguieron varias especies en base a diferencias morfológicas considerando que no fueran atribuibles a dimorfismo sexual o variación discontinua intraespecífica (Wyatt \& Foster 1989, Thayer 1992). En sólo un género de Tenebrionidae (Praocis) los especímenes mostraron variación fenotípica sugiriendo más de una especie, pero sin concordar con los datos de distribución geográfica (Vidal 2000) de las especies putativas por lo que se le consideró un solo taxón usando un criterio conservativo. Por ello, los insectos determinados fueron tratados como especies aun cuando no se estableciera su nombre binomial. Muestras representativas de los insectos colectados fueron etiquetadas para su reconocimiento e ingresadas como conjunto en la "Colección de Flora y Fauna Profesor Patricio Sánchez Reyes”, Sala de Sistemática, Pontificia Universidad Católica de Chile, Santiago (código único: SSUC-6915).

El número de especies y de individuos de insectos capturados fueron analizados mediante esta- dística no paramétrica, usando la prueba de Kruskal-Wallis para comparación entre las cuatro localidades y la prueba de Friedman para comparación entre los tres niveles intermareales. El uso de estas pruebas obedeció tanto al bajo número y falta de normalidad de los datos, para preservar la estructura original evitando transformaciones, como a su potencia que es comparable a la de sus respectivos equivalentes paramétricos y mayor que otras pruebas no paramétricas similares (ver Siegel \& Castellan 1988). Para validar la comparación de riqueza de especies entre las localidades de estudio, se efectuó un análisis de rarificación ("rarefaction"; ver Gotelli \& Graves 1996) mediante 1.000 iteraciones con el programa EcoSim (Gotelli \& Entmisnger 2001), tomando como referencia la localidad con mayor número de especies y de individuos (Cocholgüe). Así se estimó la riqueza esperada de especies en Cocholgüe si se hubiera registrado el mismo número total de individuos que en las demás localidades. Por otra parte, se evaluó si la representación de las especies en las cuatro localidades equivalía a la esperable por un proceso aleatorio. A partir de la matriz de abundancia (especies $\mathrm{x}$ localidades) se generó una matriz de valores simulados obtenidos en 10.000 aleatorizaciones de los observados. Mediante un estadístico de desviación de Chi-cuadrado, se comparó el ajuste de ambas matrices con los valores esperados, obteniendo la probabilidad de que lo observado fuese mayor o menor a lo esperado por azar (Gotelli \& Graves 1996, Gotelli \& Entmisnger 2001).

Con objeto de tener un indicador indirecto del modo o amplitud en que los insectos usarían el hábitat intermareal, para cada especie se registró el tipo de substrato intermareal biótico o abiótico en que se encontró, buscando asociaciones recurrentes. Esta información, más la ubicación relativa en el gradiente intermareal, podrá orientar estudios futuros para evaluar la selectividad o preferencia de una especie por determinados substratos. La asociación se evaluó en forma simple como el porcentaje de ocurrencia de un insecto sobre un tipo de substrato, expresado sobre el total de substratos distintos en que fue detectado. Por la falta de antecedentes para evaluar asociaciones particulares, los tipos de substrato fueron categorizados a posteriori, considerando sólo aquellos insectos asociados a organismos (i.e., substratos, tanto vivos como muertos o en descomposición). Se cuantificó las veces en que un insecto se encontró en un tipo de substrato, definido como un organismo o grupo de organismos. Los casos en que un mismo substrato se registró en distinta condición (vivo o muerto) fueron evaluados por separado. Los substratos y/o insectos 
no relacionados al intermareal fueron excluidos de este cálculo y se indican en Resultados cuando corresponde. No se evaluó la abundancia de cada substrato en terreno.

A partir de los datos anteriores y para el conjunto de insectos seleccionados, se obtuvieron dos estimaciones adicionales: la frecuencia global de uso de organismos vivos versus muertos sin distinguir substratos particulares, y la frecuencia de uso de cada especie de alga pero evaluando por separado su condición (viva versus muerta).

\section{RESULTADOS}

\section{Diversidad en el área de estudio}

En las cuatro localidades de muestreo se registró un total de 24 taxa de insectos, tanto de Chile como introducidos, distribuidos en cuatro órdenes y 18 familias (Tabla 1; registros por localidad y nivel intermareal). Para 11 taxa se logró determinar su posición taxonómica a nivel de género (5) o especie (6), y sólo a nivel de familia a los 13 restantes.

No obstante, no se descarta que un examen taxonómico posterior pueda aumentar el número de especies, ya que dos Tenebrionidae mostraron alguna variación en color (Nycterinus sp., de negro a tonos más claros) o patrón de coloración (Praocis sp., negro uniforme o con líneas blanquecinas difusas). Por otra parte, la simulación aleatoria de la matriz de abundancia mostró que los valores observados (i.e., la distribución de números de especies e individuos entre las cuatro localidades) eran consistente y significativamente menores a los esperados por azar $(\mathrm{P}=0,00001$; tamaño de efecto estandarizado: 4,52). En las

TABLA 1

Insectos registrados en cuatro localidades (BB: desembocadura río Bío-Bío; CO: Cocholgüe; CT: Caleta Tumbes; PP: Punta de Parra) y tres niveles intermareales (A: alto; M: medio; B: bajo) en la costa de Concepción, Chile

Insects recorded in four localities (BB: mouth of Bío-Bío river; CO: Cocholgüe; CT: Caleta Tumbes; PP: Punta de Parra) at three intertidal levels (A: high; M: mid; B: low) on the coast of Concepción, Chile

\begin{tabular}{|c|c|c|}
\hline Taxón (Orden, Familia, especie) & Localidad & Nivel mareal \\
\hline \multicolumn{3}{|l|}{ Coleoptera } \\
\hline Anthicidae, no determinado & $\mathrm{BB}, \mathrm{CO}, \mathrm{PP}$ & M \\
\hline Carabidae, Metius sp. & $\mathrm{BB}, \mathrm{CO}, \mathrm{CT}, \mathrm{PP}$ & $\mathrm{M}, \mathrm{B}$ \\
\hline Coccinellidae, Eriopis connexa & $\mathrm{BB}, \mathrm{CO}, \mathrm{PP}$ & M \\
\hline Curculionidae, Cylidrorhinus sp. & $\mathrm{BB}$ & M \\
\hline Curculionidae, Rhyephenes maillei & $\mathrm{CO}$ & M \\
\hline Histeridae, no determinado & $\mathrm{CO}$ & A \\
\hline Scarabaeidae, Brachysternus sp. & $\mathrm{CO}$ & A \\
\hline Staphylinidae, no determinado 1 & $\mathrm{CO}$ & $\mathrm{A}, \mathrm{M}, \mathrm{B}$ \\
\hline Staphylinidae, no determinado 2 & $\mathrm{CO}$ & $\mathrm{A}, \mathrm{M}, \mathrm{B}$ \\
\hline Staphylinidae, no determinado 3 & $\mathrm{BB}$ & $\mathrm{M}$ \\
\hline Staphylinidae, no determinado 4 & $\mathrm{BB}, \mathrm{PP}$ & M \\
\hline Tenebrionidae, Nycterinus sp. & $\mathrm{BB}, \mathrm{CO}$ & A, M \\
\hline Tenebrionidae, Phalerisida maculata & $\mathrm{BB}, \mathrm{CO}, \mathrm{PP}$ & M \\
\hline Tenebrionidae, Praocis sp. & $\mathrm{BB}$ & $\mathrm{A}, \mathrm{M}$ \\
\hline \multicolumn{3}{|l|}{ Dermaptera } \\
\hline Forficulidae, Forficula auricularia & $\mathrm{CO}$ & A \\
\hline \multicolumn{3}{|l|}{ Diptera } \\
\hline \multicolumn{3}{|c|}{ Chironomidae, Sub Familia Orthocladiinae } \\
\hline Tribu Clunionini, no determinado & $\mathrm{CO}$ & $\mathrm{B}$ \\
\hline Muscidae, no determinado & $\mathrm{BB}, \mathrm{CO}, \mathrm{CT}$ & $\mathrm{B}, \mathrm{M}$ \\
\hline Stratyomidae, Beridops maculipennis & $\mathrm{BB}$ & M \\
\hline Syrphidae, no determinado & $\mathrm{CO}$ & M \\
\hline Tabanidae, Scaptia lata & $\mathrm{CT}$ & $\mathrm{B}$ \\
\hline Tachinidae, no determinado & $\mathrm{BB}, \mathrm{CO}, \mathrm{CT}$ & $\mathrm{M}, \mathrm{B}$ \\
\hline Tipulidae, no determinado & $\mathrm{CO}$ & $\mathrm{B}$ \\
\hline Zapromysidae, no determinado & $\mathrm{BB}$ & $\mathrm{M}$ \\
\hline \multicolumn{3}{|l|}{ Hymenoptera } \\
\hline Megachilidae, no determinado & $\mathrm{CO}$ & M \\
\hline
\end{tabular}


10.000 iteraciones, $99,99 \%$ de las veces la desviación (distancia de Chi-cuadrado) de las observaciones respecto a la matriz aleatoria fue mayor que la desviación de los valores en la matriz simulada. Por ello, y dado que sólo se muestreó una vez por localidad y se registró un número alto de familias con sólo 1,3 especies por familia en promedio, es muy probable que muestreos más intensivos en tiempo y espacio detecten una mayor cantidad de especies e individuos.

\section{Diversidad por localidad}

Las cuatro localidades estudiadas fueron altamente variables en su número total de individuos y especies de insectos, respectivamente: 77 y 16 en Cocholgüe, 51 y 13 en la desembocadura del río Bío-Bío, 12 y cinco en Punta de Parra, ocho y cuatro en Caleta Tumbes. Dado que el esfuerzo y diseño muestral fue el mismo en todos los casos, las diferencias no se deberían a sesgos de muestreo. El análisis de rarificación mostró que la riqueza esperada de especies en Cocholgüe (lugar con máxima riqueza y abundancia), si se hubiera registrado el número de individuos observado en otra localidad, correspondería a (me- dia \pm intervalo de confianza $95 \%$ ): $11,14 \pm 2,51$ respecto a la desembocadura del río Bío-Bío, 5,13 $\pm 2,41$ respecto a Punta de Parra, y 4,02 $\pm 2,19$ respecto a Caleta Tumbes. No hubo diferencias significativas $(\alpha=0,05)$ entre la riqueza esperada en Cocholgüe para muestras equivalentes a las de otras localidades, sugiriendo que, aun cuando la diversidad observada es menor a la esperada y sólo es comparable bajo condiciones de muestreo similares, la divergencia en diversidad entre las localidades podría reflejar adecuadamente la situación real.

Las Fig. 1 y 2 muestran respectivamente la riqueza de insectos y el número de individuos registrados en cada localidad para los tres niveles intermareales muestreados, notándose una tendencia general a un mayor valor de ambas variables en la zona intermareal media. Pese a ello, no hubo diferencias estadísticas entre localidades tanto en la riqueza de especies de insectos (prueba de Kruskal-Wallis; K-W = 3,00; g.l. = 3; P = $0,262)$ como en el número de individuos $(\mathrm{K}-\mathrm{W}=$ 3,$00 ;$ g.l. $=3 ; \mathrm{P}=0,392$ ). Sin embargo, entre niveles mareales sí se registró una diferencia en riqueza de especies (prueba de Friedman; $\mathrm{X}^{2}=$ $4,00 ;$ g.l. $=1 ; \mathrm{P}=0,046)$ asociada a una mayor diversidad en el intermareal medio, aunque no hubo diferencias en el número de individuos (prue-

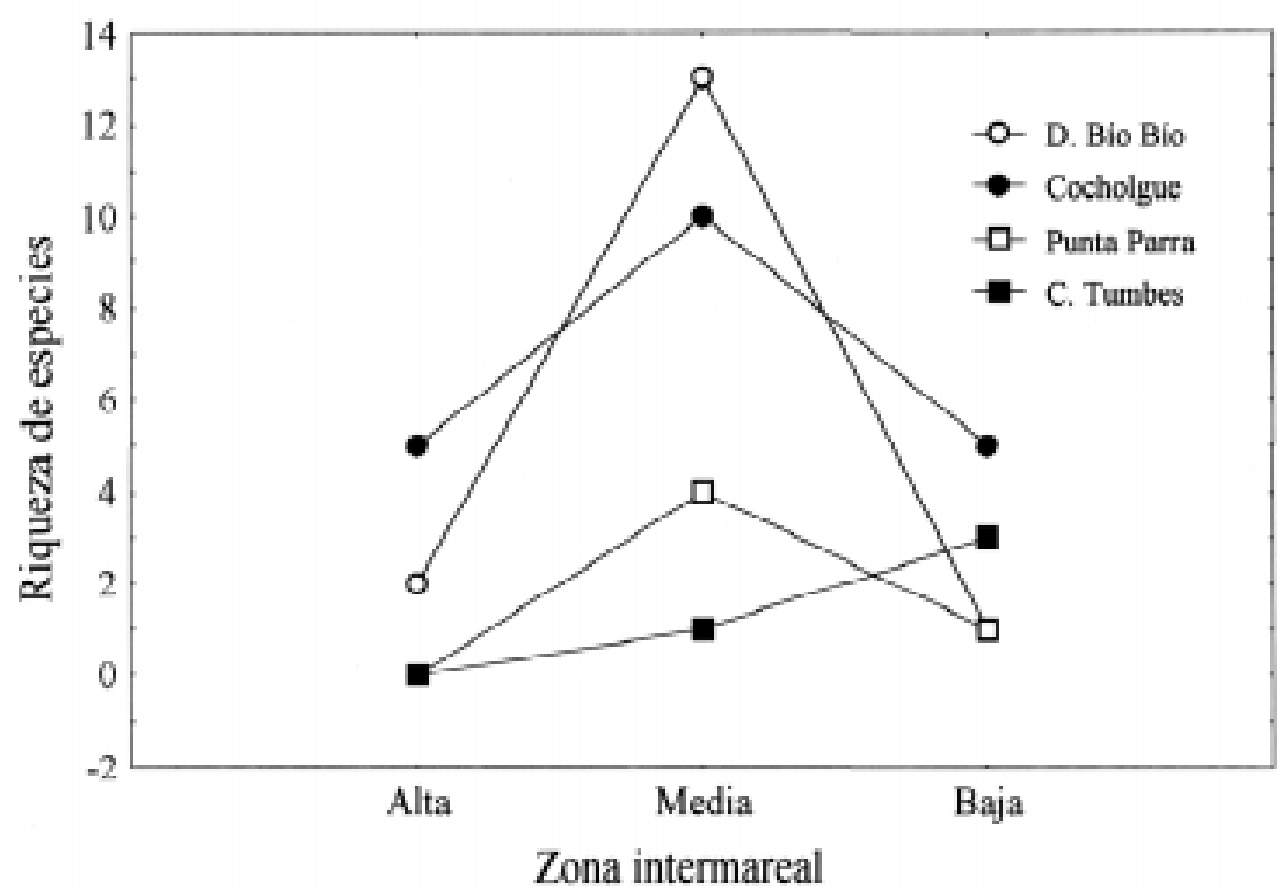

Fig. 1: Riqueza de insectos registrada en el hábitat intermareal de cuatro localidades en la costa de Concepción. Las estaciones de muestreo fueron agrupadas en tres zonas verticales para cada localidad. Insect richness recorded at the intertidal habitat of four localities from the coast of Concepcion. Sampling stations were pooled into three vertical zones for each locality. 
ba de Friedman; $\mathrm{X}^{2}=4,50 ;$ g.l. $\left.=2 ; \mathrm{P}=0,105\right)$. La mayor diversidad de insectos en el intermareal medio aparentemente no sería casual, y puede estar relacionada a la presencia en esa zona de las algas más frecuentes y abundantes en hábitats rocosos (ver Tablas 2 y 3 ).

\section{Asociaciones entre insectos y especies intermareales}

Los insectos capturados representan una colección heterogénea de especies. Sólo 4,2 \% (un taxon: Metius sp.) estuvo en las cuatro localidades, $20,8 \%$ en tres, $16,7 \%$ en dos, y $58,3 \%$ en sólo una localidad (ver Tabla 2). Además los taxa divergían claramente en su grado de vinculación al hábitat intermareal. Una fracción de ellos son típicos de hábitats exclusivamente terrestres, por lo que en principio podrían considerarse visitantes ocasionales o casuales, o sin relevancia particular en el ambiente marino. Entre ellos están Brachysternus sp., Nycterinus sp. y Praocis sp., encontrados asociados a plantas terrestres o sus restos, además del gorgojo de la madera Rhyephenes maillei encontrado en restos de tron- cos. También se registró a Metius sp. principalmente en substratos no biológicos y desechos, a especies como Forficula auricularia asociada a desechos o restos de troncos, y a Eriopis connexa asociada a macroalgas café varadas. Otros taxa como Scaptia lata ("colihuacho") o especies de Megachilidae y Syrphidae volaban desplazándose a través de toda la zona intermareal, probablemente en busca de presas o alimento o sólo en tránsito. Algunos taxa como el curculiónido Cylidrorhinus sp. estarían usando sectores del intermareal que mantienen condiciones propiamente terrestres o hay individuos o pequeños parches de plantas terrestres (especialmente en la zona alta de ecotono), y otros como Rhyephenes parecen aprovechar desechos o restos de vegetación terrestre como hábitat o alimento

En contraste, otras especies estuvieron asociadas consistentemente a substratos orgánicos intermareales (Tabla 2), encontrándose principalmente entre o bajo frondas de los géneros de algas más abundantes del intermareal rocoso (Gelidium, Mastocarpus, Mazaella y Ulva), o en macroalgas café varadas en arena o rocas (Durvillaea, Macrocystis). Mientras los individuos de Chironomidae, Muscidae y Tipulidae sólo

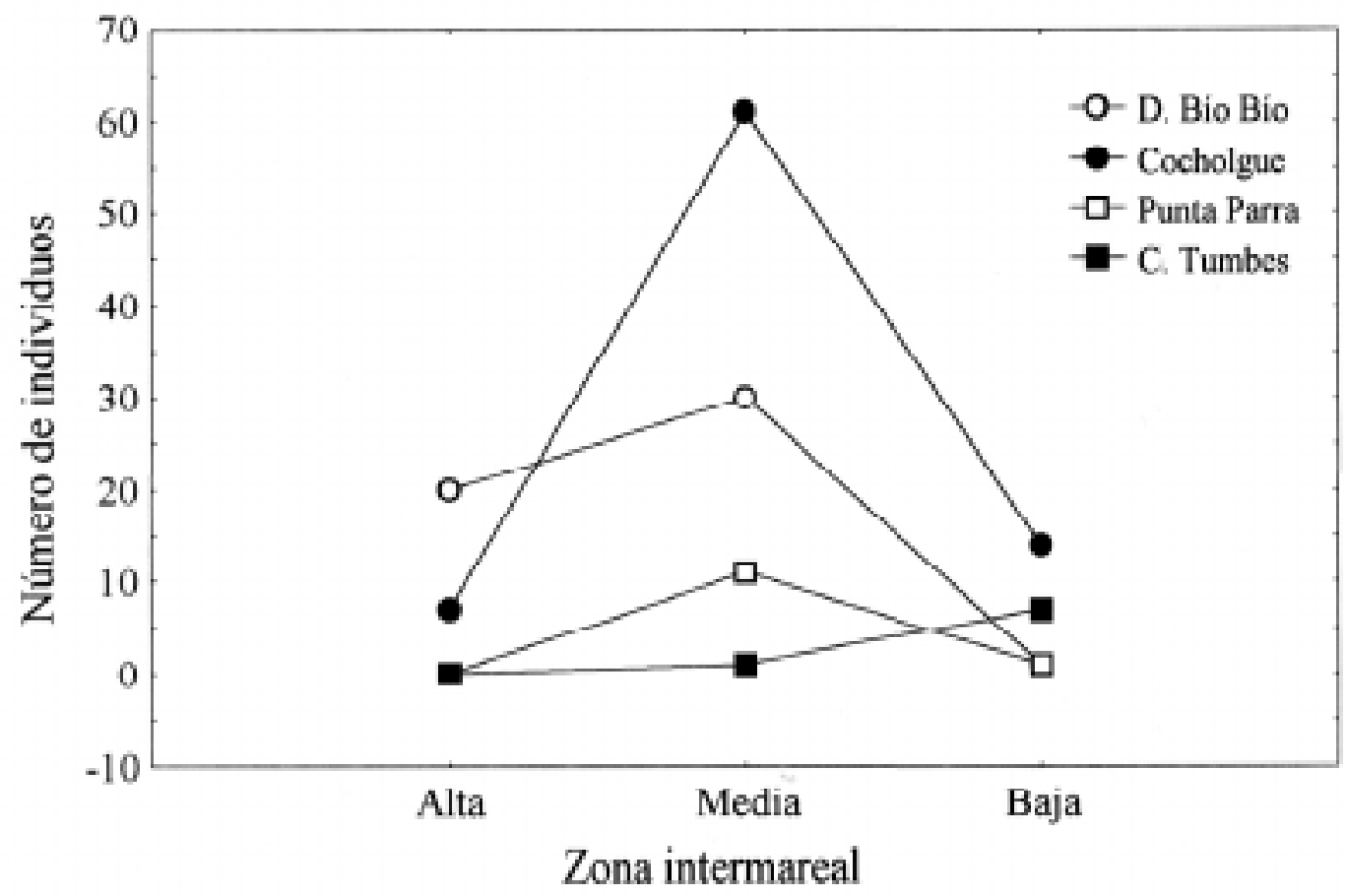

Fig. 2: Número de individuos (insectos adultos) registrados en el hábitat intermareal de las cuatro localidades de estudio. Las estaciones de muestreo fueron agrupadas por zona intermareal para cada localidad.

Number of individuals (adult insects) recorded at the intertidal habitat of the four study localities. Sampling stations were pooled by intertidal zone for each locality. 
TABLA 2

Asociaciones consistentes detectadas en las localidades de estudio (agrupadas) entre insectos (excluyendo especies ocasionales) y substratos orgánicos vivos y muertos. Valores: porcentajes de ocurrencia sobre un tipo de substrato (conjunto de algas o animales; ver Métodos). Las especies de algas se indican en Tabla 3

Recurrent associations detected in the study localities (pooled) between insects (excluding occasional species) and living or dead organic substrata. Values: percentage occurrence on a given substratum (group of algal or animal genera; see Methods). Algal species listed in Table 3

\begin{tabular}{ll}
\hline Taxón & Substrato biológico \\
\hline Anthicidae & Algas muertas (33,3\% en Gelidium-Mastocarpus, 33,3\% en Macrocystis-Durvillaea) \\
Beridops maculigennis & Algas muertas (100\% en Macrocystis-Durvillaea) \\
Chironomidae & Algas vivas (100\% en Mazaella-Lessonia) \\
Eriopis connexa & Algas muertas (66,6\% en Macrocystis, 33,3\% en Gelidium) \\
Metius sp. & Algas muertas (25\% en Durvillaea) y vivas (50 \% en Gelidium-Mastocarpus) \\
Muscidae & Algas vivas (50\% en Lessonia-Mazaella, 50\% en Ulva-Mastocarpus) \\
Phalerisida maculata & Algas muertas (100\% en Macrocystis) \\
Staphylinidae & Algas muertas (28,6\% en Macrocystis), algas vivas (42,9\% en Ulva-Chondracanthus- \\
& Mastocarpus), restos animales (28,6 \% en Pyura-crustáceos) \\
Tachinidae & Algas muertas (33,3\% en Macrocystis-Durvillaea) y vivas (66,6 \% en Mazaella- \\
& Mastocarpus) \\
Tipulidae & Algas vivas (100\% en Mazaella) \\
Zapromysidae & Algas muertas (100\% en Macrocystis-Durvillaea) \\
\hline
\end{tabular}

se asociaron a algas vivas, otros como Phalerisida maculata y varios Staphylinidae y dípteros se encontraron recurrentemente en organismos muertos o en descomposición en estado adulto y larval. Es sugerente que depredadores como Eriopis connexa, sin conexión con el ambiente marino, sólo se detectaron sobre algas en descomposición donde se concentran adultos y larvas de otros insectos.

La Tabla 3 muestra la frecuencia con que los taxa de la Tabla 2 usaron distintos substratos orgánicos, categorizados en general como vivos o muertos y en particular por tipo de alga. Los insectos estaban asociados básicamente a las al-

TABLA 3

Uso general de substratos orgánicos por los taxa de insectos basado en Tabla 2, expresado como porcentaje $(\%)$ de los taxa $(\mathrm{n}=11)$ asociado a un substrato particular: (A) por tipo de substrato; (B) por género de algas, separadamente para algas vivas y muertas

Generalized use of organic substrata by insect taxa on the basis of Table 2, expressed as the percentage (\%) of taxa $(n=11)$ associated to a particular substratum: (A) by substratum type; (B) by algal species, separately for live and decaying algae

\begin{tabular}{|c|c|c|}
\hline (A) Tipo de substrato & \multicolumn{2}{|c|}{ Frecuencia de uso (\%) } \\
\hline Algas vivas & \multicolumn{2}{|c|}{54,5} \\
\hline Algas muertas/en descomposición & \multicolumn{2}{|c|}{72,7} \\
\hline Restos animales & \multicolumn{2}{|c|}{9,1} \\
\hline (B) Especies de algas & Vivas & Muertas/en descomposición \\
\hline Chondracanthus chamissoi & 9,1 & 0 \\
\hline Durvillaea antarctica & 0 & 45,5 \\
\hline Gelidium sp. & 9,1 & 18,2 \\
\hline Lessonia nigrescens & 18,2 & 0 \\
\hline Macrocystis pyrifera & 0 & 63,6 \\
\hline Mazaella laminarioides & 36,4 & 0 \\
\hline Mastocarpus papillatus & 36,4 & 9,1 \\
\hline Ulva lactuca & 18,2 & 0 \\
\hline
\end{tabular}


gas (en mayor proporción a plantas muertas), que en términos relativos representaron el 93,3\% de los substratos orgánicos. La frecuencia de uso de las algas fue contrastante sugiriendo una importancia diferencial para los insectos, pero el mayor o menor uso de cada especie dependió de su estado orgánico y del hábitat en que se encontró. En frecuencias relativas, Macrocystis pyrifera y Durvillaea antarctica (varadas) representaron el $80 \%$ del uso de algas muertas, y Mastocarpus papillatus y Mazaella laminarioides (comunes en el intermaeal rocoso) el $57,1 \%$ de algas vivas.

\section{DISCUSIÓN}

La diversidad registrada fue relativamente baja, pero puede representar una fracción de la real por razones taxonómicas y muestrales. Por una parte, taxa como Praocis y Nycterinus mostraron variación fenotípica que en análisis posteriores podría llevar a distinguir más de una especie por género, aunque los datos disponibles no avalaron esta conclusión por ahora. Mientras algunos individuos de Praocis sp. de color negro uniforme podrían corresponder a $P$. pilula, distribuida de Talca a Puerto Montt, otros con líneas difusas de color blanquecino fueron similares a $P$. chilensis, que sin embargo está descrita sólo para la zona norte (ver Vidal 2000). También Nycterinus sp. mostró alguna variación continua en color, pero no se logró resolver si correspondía a más de una especie. No obstante, la falta de información sobre insectos acuáticos es un problema por el momento insalvable que dificulta alcanzar determinaciones a nivel específico incluso en estudios exhaustivos (e.g., Massaferro et al. 2002). Por otra parte, el muestreo se realizó en una sola época por limitaciones logísticas y porque se planteó como preliminar para otros estudios, pero una evaluación completa de la diversidad existente requeriría un alto esfuerzo temporal tomando un ciclo estacional completo o al menos las estaciones cálidas, y contemplando muestreos con una frecuencia horaria. De hecho, el período de emergencia de adultos en otras costas (Endrab 1976a, Cheng \& Collins 1980, Neumann 1986, Saigusa \& Akiyama 1995) es altamente específico, y algunos insectos se encuentran sólo en ciertas estaciones del año y/o a ciertas horas de la mañana o la noche, incluso variando el momento según la estación.

Adicionalmente, la prueba de aleatorización mostró que es esperable una mayor riqueza efectiva de insectos, pero el análisis de rarificación también sugirió que las diferencias observadas entre localidades no se deberían a sesgos de muestreo. Es probable que esta variación espacial se deba a una calidad diferencial de hábitat para los insectos y que refleje una situación real de sitios pobres versus ricos en diversidad, aspecto que amerita más exploración ya que se ha mostrado que puede utilizarse como un indicador efectivo de calidad ambiental de hábitats costeros (Williams $2001^{2}$ ).

En general, nuestros resultados sugieren que los insectos como grupo pueden tener una participación ecológica importante en el sistema intermareal. Si bien una fracción de los taxa no tuvo ninguna relación con este hábitat (e.g., Rhyephenes maillei, taladrador de madera considerado plaga forestal; Loewe et al. 1998, Parra et al. 2001), otros como Chironomidae y Staphylinidae sí integrarían la membrecía, lo que se puede confirmar investigando su ciclo de vida. En este registro preliminar no se detectó ningún insecto estrictamente marino, como los quironómidos del género Pontomyia (Soong et al. 1999) que han perdido su capacidad de vuelo y reducido sus apéndices locomotores, pero su presencia no se puede descartar aún. No obstante, el espectro posible de insectos habitantes del intermareal es amplio, destacando Coleoptera y Diptera como usuarios frecuentes de ambientes marinos (excepto océano abierto; Williams \& Feltmate 1992). Entre los Coleoptera encontrados en este estudio, la familia Staphylinidae, de la que se registró cuatro taxa, incluye varios representantes intermareales (Sawada 1955), destacando el género Bledius con ejemplos conductuales excepcionales (e.g., B. spectabilis) asociados al cuidado parental de las larvas y a la construcción de microhábitats complejos que utilizan sistemas de presión para mantener refugios aislados del agua de mar. Entre los Diptera, miembros de las familias Ceratopogonidae, Chironomidae, Culicidae, Dolichopodidae, Ephydridae, Psychodidae, Tabanidae y Tipulidae usan regularmente el intermareal rocoso y sus pozas de marea además del intermareal arenoso (Williams \& Feltmate 1992, Narf 1997). El único Chironomidae registrado en este estudio perteneció a la tribu Clunionini, donde también se encuentra Clunio marinus, habitante de las costas de Europa occidental, y una de las pocas especies intermareales estrictas que ha llegado a sincronizar finamente sus patrones de reproducción y uso de

${ }^{2}$ WILLIAMS KS (2001) Insect densities reflect environmental quality in coastal habitats: three case studies. Annual Meeting Entomological Society of America, San Diego, California. 
hábitat con los ritmos mareales, similar al género Pontomyia (ver Neumann 1976, Soong et al. 1999). Por ello no sorprendería que un análisis posterior de los dípteros capturados en Concepción resultara en la identificación de taxa nuevos o emparentados con C. marinus, ya que las especies marinas de este grupo están siendo encontradas y descritas cada vez con mayor frecuencia (e.g., Murray 1999, Murray \& Hughes 2000).

Respecto al potencial de investigación, consideramos que el solo hecho de establecer la membrecía intermareal o el grado de conexión de los insectos con el sistema o sus especies ofrece varias perspectivas emergentes a nivel autoecológico, poblacional, comunitario y ecosistémico, tanto en el ámbito entomológico como en el marco de la ecología intermareal.

Un aspecto prioritario es el estudio taxonómico detallado de la entomofauna litoral, especialmente de los estados larvales cuya determinación presenta la mayor dificultad. Por otro lado, uno de los roles potenciales más importantes sería el consumo de algas vivas y la degradación de restos orgánicos vegetales o animales por parte de los estados larvales. Sólo recientemente se comenzó a evaluar el intercambio de materia y energía entre sistemas marinos litorales en Chile (Rodríguez 1999), pero la importancia y magnitud de los aportes provenientes del intermareal han sido poco estudiados. En este contexto, varios de los insectos adultos registrados señalan la presencia de sus larvas como componentes regulares del sistema intermareal (e.g., Neumann 1976, Soong et al. 1999). Estas larvas podrían estar entre los organismos más eficientes como procesadores de materia, y como contribuyentes al flujo de nutrientes hacia el mismo intermareal como desde el intermareal al submareal.

Otra perspectiva interesante viene de las asociaciones detectadas entre insectos y la cobertura algal intermareal. De acuerdo a la literatura, varios insectos prefieren substratos duros con algas para depositar sus huevos y servir de hábitat, alimento o refugio para sus larvas (Cheng \& Collins 1980, Neumann 1986, Saigusa \& Akiyama 1995). Sin embargo la misma literatura es poco orientadora sobre la especificidad de las relaciones, ya que rara vez se identifican las algas y más bien se describen como texturas o formas (filamentos, matas, etc.). En este estudio, los insectos tuvieron mayor asociación con algas abundantes o frecuentes en el intermareal rocoso, en especial de la zona media, sugiriendo asociaciones de carácter amplio pero sin descartar posibles relaciones interespecíficas entre insectos y algas, o entre insectos en cuanto a sus patrones de uso de substratos. El corto tiempo generacional de los insectos permitiría establecer de forma simple sus patrones de actividad y forrajeo (e.g., ver Manica et al. 2000, McMeechan et al. 2000), revelando su grado de interacción con algas marinas, o también procesos indirectos ya que por ejemplo algunas larvas de dípteros encontradas en algas no se alimentan de ellas sino de hongos asociados al proceso de descomposición (V. Jerez comunicación personal).

En relación a lo anterior, un aspecto potencialmente relevante es la susceptibilidad de los insectos a compuestos o metabolitos secundarios producidos por las algas, lo que podría generar un uso diferencial de ellas. En este sentido, es sugerente que las algas asociadas con mayor frecuencia a los insectos sean rodófitas o clorófitas (e.g., Ulva, Mastocarpus, Mazaella) que no son conocidas por contener compuestos que afectan negativamente a los consumidores. Aunque tal fenómeno es frecuente en feófitas (e.g., Duffy \& Hay 1990, Hay 1996), que son comunes en el litoral de Concepción (e.g., Desmarestia, que concentra $\mathrm{H}_{2} \mathrm{SO}_{4}$ en sus tejidos; Pelletreau \& Muller-Parker 2002), varias rodófitas chilenas poseen compuestos bioactivos (e.g., San Martín et al. 1983, 1991) que con frecuencia tienen actividad insecticida (e.g., San Martín et al. 1991, Argandoña et al. 2000).

Otras interacciones potenciales involucran a depredadores terrestres como el coleóptero Eriopis connexa, especie muy común conocida como depredadora de áfidos (e.g., Martos et al. 1992, Grez \& Villagrán 2000), que podría estar aprovechando parches de larvas detritívoras que degradan restos algales. Además, especies como el dermáptero Forficula auricularia, que en primera instancia se consideró ocasional, es normalmente eurifágica pero en hábitats silvestres actúa como depredadora generalista de insectos (Vidal 2000). Casos de este tipo podrían representar cadenas laterales de interacción conectadas al sistema intermareal, aunque se desconoce su estabilidad y relevancia.

Por todo lo anterior, el rol de los insectos en ambientes marinos es un tópico de interés creciente a nivel mundial (la Entomological Society of America realizó su primer simposio sobre insectos marinos sólo el año 2001), y es esperable que pronto dejen de ser el componente olvidado en la ecología intermareal.

\section{AGRADECIMIENTOS}

Agradecemos sinceramente a Danilo Cepeda (Universidad de Chile) y Juan Moroni (Servicio Agrícola y Ganadero) por su tiempo y colaboración 
especialmente en la determinación taxonómica de insectos, a Vivian Jerez y Luis Parra (Universidad de Concepción) por su buena disposición ante nuestra ignorancia entomológica, a Patricio Zavala (Pontificia Universidad Católica de Chile; grande Pato!) por facilitarnos el ingreso de los especímenes a una colección sistemática, y a Marcelo Lagos, Gonzalo Padilla, Marcelo Silva y María Valderrama (Universidad Católica de la Santísima Concepción) por su asistencia en terreno.

\section{LITERATURA CITADA}

AVELLANAL MH, E JARAMILLO, E NAYLOR \& F KENNEDY (2000) Orientation of Phalerisida maculata Kulzer (Coleoptera, Tenebrionidae) in sandy beaches of the Chilean coast. Journal of Experimental Marine Biology and Ecology 247: 153-167.

ARGANDOÑA V, T DEL POZO, A SAN MARTIN \& J ROVIROSA (2000) Insecticidal activity of Plocamium cartilagineum monoterpenes. Boletín de la Sociedad Chilena de Química 45: 371-376.

BARNES RSK \& RN HUGHES (1988) An introduction to marine ecology. Second edition. Blackwell Scientific Publications, Oxford, United Kingdom. $x+351$ pp.

CHENG L (ed) (1976) Marine insects. North-Holland Publishing Company, Amsterdam, The Netherlands. $581 \mathrm{pp}$.

CHENG L \& JD COLLINS (1980) Observations on behavior, emergence and reproduction of the marine midges Pontomya (Diptera: Chironomidae). Marine Biology 58: 1-5.

COMSTOCK JH (1977) An introduction to entomology. Ninth edition. Hodder \& Staughton, London, United Kingdom. xiv +919 pp.

CSIRO (1979) The insects of Australia. Third edition. Melbourne University Press, Canberra, Australia. xiii $+1029 \mathrm{pp}$.

DUFFY JE \& ME HAY (1990) Seaweed adaptations to herbivory. Bioscience 40: 368-375.

ENDRAb U (1976a) Physiological adaptations of a marine insect. I. The temporal programming of development. Marine Biology 34: 361-368.

ENDRAb U (1976b) Physiological adaptations of a marine insect. II. Characteristics of swimming and sinking egg-masses. Marine Biology 36: 47-60.

EVENHUIS NL, ed (1999) Catalog of the Diptera of the Australasian and Oceanian Regions, web version. Bishop Museum, Honolulu, Hawaii. http:// hbs.bishopmuseum.org/aocat/aocathome.html

GOTELLI NJ \& GL ENTSMINGER (2001) EcoSim: Null models software for ecology. Versión 7.0, Acquired Intelligence Inc. \& Kesey-Bear.

GOTELLI NJ \& GR GRAVES (1996) Null models in ecology. Smithsonian Institution Press, Washington, District of Columbia. 368 pp.

GREZ AA \& P VILLAGRÁN (2000) Effects of structural heterogeneity of a laboratory arena on the movement patterns of adult Eriopis connexa and Hippodamia variegata (Coleoptera: Coccinellidae). European Journal of Entomology 97: 563-566.
HAY ME (1996) Marine chemical ecology: What's known and what's next? Journal of Experimental Marine Biology and Ecology 200: 103-134.

JARAMILLO E, MH AVELLANAL, M GONZÁLEZ \& F KENNEDY (2000) Locomotor activity of Phalerisida maculata Kulzer (Coleoptera, Tenebrionidae) on Chilean sandy beaches. Revista Chilena de Historia Natural 73: 67-77.

LIZARRALDE M (1998) Ephydridae. En: Morrone JJ \& S Coscaron (eds) Biodiversidad de artrópodos argentinos. Una perspectiva biotaxonómica, Ediciones Sur, La Plata, Argentina. viii +599 pp.

LOEWE V, M TORAL, G PINEDA, C LÓPEZ \& E URQUIETA (1998) Monografía de lenga: Nothofagus pumilio. Corporación Nacional Forestal, Instituto Forestal, Fundación para la Innovación Agraria, Santiago, Chile. 103 pp. + anexos

MANICA A, FK MCMEEHAN \& WA FOSTER (2000) Orientation in the intertidal salt marsh collembolan Anurida maritima. Behavioral Ecology and Sociobiology 47: 371-375.

MARTÍNEZ DR, DA LÓPEZ, ML DEL VILLAR, FR FIGUEROA \& HF GAJARDO (1986) Depredación de organismos intermareales por roedores. Biota (Chile) 2: 87-90.

MARTOS A, A GIVOVICH \& HM NIEMEYER (1992) Effect of DOMBOA, an aphid resistance factor in wheat, on the aphid predator Eriopis connexa (Germar) (Colleoptera: Coccinellidae). Journal of Chemical Ecology 18: 469-479.

MASSAFERRO J, SJ BROOKS \& KA JACKSON (2002) Estudio preliminar de la distribución y composición de las comunidades de quironómidos (Diptera: Chironomidae) en el parque nacional Laguna San Rafael $\left(46^{\circ} \mathrm{S}\right)$, Chile. Boletín del Museo Nacional de Historia Natural (Chile) 51: 123-134.

McMEECHAN FK, A MANICA \& WA FOSTER (2000) Rhythms of activity and foraging in the intertidal insect Anurida maritima : coping with the tide. Journal of the Marine Biological Association of the United Kingdom 80: 189-190.

MERRIT RW, DW WEBB \& EI SCHLINGER (1996) Aquatic Diptera. Part Two. Pupae and adults of aquatic Diptera. En: Merrit RW \& Cummins KW (eds) An introduction to the aquatic insects of North America: 515-548. Third edition. Kendall/Hunt Publishing Company, Dubuque, Iowa.

MURRAY DA (1999) Two marine coastal-dwelling Chironomidae (Diptera) new to the fauna of Iceland: Telmatogeton japonicus Tokunaga (Telmatogetoninae) and Clunio marinus Haliday (Orthocladiinae). Bulletin of the Irish Biogeographical Society 23: 89-91.

MURRAY DA \& S HUGHES (2000) New records of marine Chironomidae (Diptera) from Madeira. En: Hoffrichter $\mathrm{O}$ (ed) Late $20^{\text {th }}$ century research on Chironomidae: 575-588. Shaker Verlag, Achen, Germany.

NARF R (1997) Midges, bugs, whirligigs and others: The distribution of insects in Lake "U-Name-It». Lakeline. North American Lake Management Society 16-17: 57-62. 
NAVARRETE SA \& JC CASTILLA (1993) Predation by Norway rats in the intertidal zone of central Chile. Marine Ecology Progress Series 92: 187-199.

NEUMANN D (1976) Adaptations of chironomids to intertidal environments. Annual Review of Entomology 21: 387-414.

NEUMANN D (1986) Diel eclosion rhythm of a sublittoral population of the marine insect Pontomyia pacifica. Marine Biology 90: 461-465.

PARRA P, M GONZÁLEZ, JC VALENCIA \& J FAÚNDEZ (2001) Manual para la certificación sanitaria forestal. INFOR, Manual No. 38, Santiago, Chile. 45 pp.

PELLETREAU KN \& G MULLER-PARKER (2002) Sulfuric acid in the phaeophyte alga Desmarestia munda deters feeding by the sea urchin Strongylocentrotus droebachiensis. Marine Biology 141: 1-9.

PEÑA LE (1992) Introducción a los insectos de Chile. Editorial Universitaria, Santiago, Chile. $256 \mathrm{pp}$.

RAFFAELLI D \& S HAWKINS (1996) Intertidal ecology. Chapman \& Hall, London, United Kingdom. $\mathrm{x}+356$ pp.

RODRÍGUEZ S (1999) Subsidios tróficos en ambientes marinos: la importancia de las macroalgas pardas a la deriva como fuente exógena de recursos alimentarios para el erizo Tetrapygus niger (Echinodermata: Echinoidea) en el intermareal rocoso de la costa de Chile central. Tesis doctoral, Departamento de Ecología, Pontificia Universidad Católica de Chile, Santiago, Chile. 117 pp.

ROSE MD \& GA POLIS (1998) The distribution and abundance of coyotes: the effects of allochthonous food subsidies from the sea. Ecology 79: 998-1007.

SAIGUSA M \& T AKIYAMA (1995) The tidal rythm of emergence, and the seasonal variation of this synchrony, in an intertidal midge. Biological Bulletin 188: $166-178$.
SAN MARTÍN A, J ROVIROSA, O MUÑOZ, MH CHEN, RD GUMERATNE \& J CLARDY (1983) The isolation and structure determination of Chilenone $\mathrm{A}$, a putative dimer of 2-methyl-3 (2H) furanine from the marine alga Laurencia chilensis. Tetrahedron Letters 24: 4063-4066.

SAN MARTÍN A, R NEGRETE \& J ROVIROSA (1991) Insecticide and acaricide activities of polyhalogenated monoterpenes from Chilean Plocamium cartilagineum. Phytochemistry 30: 2165-2169.

SAWADA K (1955) Marine insects of the Tokara Islands VIII. Family Staphylinidae (Coeloptera). Publications of the Seto Marine Biological Laboratory 5: 81-87.

SIEGEL S \& NJ CASTELLAN (1988) Nonparametric statistics for the behavioral sciences. Second edition. McGraw-Hill Book Company, New York, New York. $\mathrm{xxiii}+399 \mathrm{pp}$

SOONG K, G-F CHEN \& J-R CAO (1999) Life history studies of the flightless marine midges Pontomya spp. (Diptera: Chironomidae). Zoological Studies 38: 466-473.

TESKEY HJ (1991) Key to families of larvae. En: Stehr WF (ed) Inmature insects, Volume 2: 708-876. Kendal/ Hund Publishing Company.

THAYER MK (1992) Discovery of sexual wing dimorphism in Staphylinidae (Coleoptera): "Omalium" flavidum, and a discussion on wing dimorphism in insects. Journal of the New York Entomological Society 100: 540-573.

VIDAL P (2000) Insectos de Chile/Chilean insects. http:/ /vidal.med.puc.cl

WILLIAMS DD \& BW FELTMATE (1992) Aquatic insects. CAB International, Oxford, United Kingdom. $\mathrm{xiii}+358 \mathrm{pp}$.

WYATT TD \& WA FOSTER (1989) Parental care in the subsocial intertidal beetle Bledius spectabilis in relation to parasitism by the Ichneumonid wasp Barycnemis blediator. Behaviour 110: 76-92.

ZAMORANO JH (1986) Rattus rattus (Rodentia, Muridae), un depredador intermareal poco conocido. Medio Ambiente (Chile) 8: 58-62.

Editor Asociado: S. Navarrete

Recibido el 12 de marzo de 2002; aceptado el 14 de octubre de 2002 